\title{
Mieux apprécier la capacité de discernement des patients
}

Fonds national suisse de la recherche scientifique (FNS)

Les médecins ont souvent des difficultés à déterminer si un patient dépressif ou atteint de démence est capable de discernement. C'est ce que révèle une étude réalisée dans le cadre du Programme national de recherche «Fin de vie» (PNR 67, sous la direction de Prof. Dr Nikola Biller-Andorno). La Commission centrale d'éthique de l'Académie Suisse des Sciences Médicales envisage désormais d'élaborer de nouveaux principes d'évaluation.

La question de la capacité de discernement des patients est déterminante dans toute thérapie médicale, car seules les personnes en mesure de comprendre, d'évaluer et de pondérer les informations du médecin peuvent donner valablement leur accord à un traitement. Si le patient n'est pas capable de discernement, la législation actuelle prévoit que le médecin doit pouvoir s'appuyer sur des directives anticipées du patient ou s'adresser à un représentant autorisé.

\section{«La très grande majorité des médecins se sent respon- sable de l'appréciation de la capacité de discernement des patients.»}

Correspondance:

Dr Manuel Trachsel

Institute for Biomedical Ethics and History of Medicine

Université de Zurich

Pestalozzistrasse 24

CH-8032 Zurich

Tél. 0786854454

manuel.trachsel[at]uzh.ch
L'incapacité de discernement apparaît souvent dans des pathologies telles que la démence ou les dépressions graves ou lors de lésions cérébrales. Justement en fin de vie, de nombreuses personnes ne sont plus capables de discernement parce qu'elles sont atteintes de maladies graves. Or, c'est précisément là que de très importantes décisions médicales complexes doivent être prises: Faut-il interrompre les mesures susceptibles de prolonger la vie? Le patient veut-il même solliciter une forme d'assistance au décès?

\section{Loin d'être triviale}

La détermination de la capacité de discernement est loin d'être triviale. La décision dépend notamment de la situation: Une personne peut par exemple passer pour capable de discernement pour des décisions du quotidien telles que le choix des vêtements ou des repas, mais comme incapable de discernement pour prendre des décisions médicales graves. En outre, les capacités cognitives, par exemple des patients atteints de maladie d'Alzheimer ou de Parkinson, peuvent varier considérablement d'un jour à l'autre. Dans un article publié récemment dans une revue spécialisée, Manuel Trachsel, maître-assistant à l'Institut d'éthique biomédicale de l'Université de Zurich, et ses collègues Helena Hermann et Nikola Biller-Andorno ont réfléchi à la manière dont les médecins pourraient apprécier la capacité de discernement malgré ces circonstances difficiles [1].

Mais qu'entendent les médecins par capacité de discernement? Dans le cadre du Programme national de recherche «Fin de vie» (PNR 67), M. Trachsel et ses collègues ont interrogé près de 760 médecins de toute la Suisse. Selon les résultats qui viennent d'être publiés [2], la très grande majorité des médecins se sent responsable de l'appréciation de la capacité de discernement des patients. Mais même parmi ceux qui se sentent «très responsables», à peine un tiers estime être suffisamment compétent pour en juger.

\section{Des règles empiriques plutôt que des principes directeurs}

Le corps médical ne dispose pas d'une définition uniforme de la notion de capacité de discernement, des principaux critères d'évaluation ni de la manière dont il juge de la capacité de discernement. La plupart des médecins ont leurs propres règles empiriques pour déterminer si un patient est capable de discernement ou non. Très rares sont ceux qui savent qu'il existe déjà divers principes directeurs pour déterminer la capacité de discernement.

L'écrasante majorité des médecins interrogés indique cependant son désir de pouvoir utiliser de tels instruments d'évaluation. Une grande majorité plaide en outre en faveur de directives claires et manifeste son intérêt pour des formations dans ce domaine. Ce souhait va désormais être réalisé: Sur la base des résultats de l'enquête, la Commission centrale d'éthique de l'Académie Suisse des Sciences Médicales (ASSM) a décidé de se pencher de plus près sur cette question à partir de l'an prochain et d'élaborer des principes directeurs pour l'évaluation de la capacité de discernement.

\section{Références}

1 Trachsel M, Hermann H, Biller-Andorno N. Cognitive Fluctuations as a Challenge for the Assessment of Decision-Making Capacity in Patients With Dementia. American Journal of Alzheimer's Disease and other Dementias. 2014; doi: 10.1177/1533317514539377.

2 Hermann H, Trachsel M, Mitchell C, Biller-Andorno N. Medical decision-making capacity: knowledge, attitudes, and assessment practices of physicians in Switzerland. Swiss Med Wkly. 2014;144:w14039. 